\title{
Molecular Dynamics/XFEM Coupling by a Three Dimensional Extended Bridging Domain with Applications to Dynamic Brittle Fracture
}

\author{
Hossein Talebi ${ }^{1}$, Mohammad Silani ${ }^{2}$, Stéphane P. A. Bordas ${ }^{3}$, Pierre Kerfriden ${ }^{3}$, Timon \\ Rabczuk $^{1}$ \\ 1 Institute of Structural Mechanics, Bauhaus-University Weimar, Marienstr. 15, D-99423 Weimar, Germany \\ 2 Department of Mechanical Engineering, Isfahan University of Technology, Isfahan, Iran 84156-83111 \\ ${ }^{3}$ Institute of Mechanics and Advanced Materials, Theoretical and Computational Mechanics, Cardiff University, Cardiff,
}

$\mathrm{UK}, \mathrm{CF} 243 \mathrm{AA}$

\begin{abstract}
We propose a method to couple a three-dimensional continuum domain to a molecular dynamics (MD) domain to simulate propagating cracks in dynamics. The continuum domain is treated by an extended finite element method, to handle the discontinuities. The coupling is based on the Bridging Domain Method (BDM) which blends the continuum and atomistic energies. The Lennard-Jones potential is used to model the interactions in the atomistic domain and the Cauchy-Born rule is used to compute the material behaviour in the continuum domain. To our knowledge, it is the first time that a three dimensional extended bridging domain method is reported. To show the suitability of the proposed method, a three-dimensional crack problem with an atomistic region around the crack front is solved. The results show that the method is capable of handling crack propagation and dislocation nucleation.
\end{abstract}

\section{Introduction}

The modelling and simulation of cracks in structure traditionally relies on energy considerations at the engineering scale: cracks propagate when a sufficient quantity of mechanical energy is available to create free surfaces in the structure. Such approaches have been successful for the prediction of failure of brittle homogeneous materials, but encounter difficulties in more complex cases, as the surface energy depends on the micro-structure of the material. Therefore, modern 
crack modelling revisits this phenomenon as a coupling between the scale of the structure and the scale of the material. However, choosing the scale at which the material failure can be accurately described is still an open issue. Recently, with the increase of computing power, researchers have tried to simply analyse the local state of the material at quantum level, in the hope than information about the local failure would not be altered by any intermediate-scale assumption. However, quantum methods are extremely expensive and can handle only systems which are still significantly smaller than engineering length scales. Molecular dynamics (MD) relies on simplifying assumptions to make computations at the atomistic scale more amenable, but are still expected to provide valuable insights into the low-scale physics of material failure. In spite of these simplifying assumptions, MD simulations can still not be performed, today, on structures of engineering relevance.

Consequently, some "model reduction" method is necessary. We are particularly interested here in methods that address the following question: "Where is the atomistic description necessary, and conversely, where is a continuum description of the structure sufficiently accurate for our purposes, and how can these two descriptions be coupled?". Consequently, this particular branch of "multiscale methods" decreases the computational expense by simply restricting the fine, atomistic treatment to areas where it is required to increase physical realism. The quasicontiuum approach Tadmor et al. [1] or the bridging domain method (BDM) S.P. Xiao [2] are example of such "concurrent multiscale methods" where the continuum description is used far way from the region of interest and the MD formulation is used at the crack front. These methods differ in the way the two descriptions are coupled, which is of tremendous importance in terms of numerical stability and accuracy. In the subset of methods comprising a variant of the Arlequin method of Ben Dhia H [3] and the bridging domain method (BDM) the continuum and MD models are coupled over a blending (or handshake) region (i.e.: the corresponding domains overlap). This algorithmic feature introduces some flexibility in the description of the coupling, which permits, to some extend, to tackle difficult numerical issues related to the dynamic field transfer between the two regions such as artificial wave reflections.

Whilst discontinuities arising from separating atomic planes in molecular dynamics do not require special treatment, dealing with evolving discontinuities in the continuum region can be challenging in a discrete setting. For example, the finite element method (FEM) is ill-suited to 
handle complicated moving fronts across which discontinuities exist, although very robust mesh generation software is now available with some very impressive results shown (inter alia, for multi-material domains in Zhang et al. [4] and for arbitrary crack problems in Cavalcante Neto et al. [5]). Since the inception of the partition of unity method (PUM) by Babuška et al. [6] in the last decade, enriched methods such as the generalised finite element method Strouboulis et al. [7], the extended finite element method [8] (XFEM) [9], the $h p$ cloud method Duarte and Oden [10] or enriched boundary element methods Simpson and Trevelyan [11, 12], enriched meshfree methods such as the enriched element free Galerkin method using extrinsic enrichment Ventura et al. 13] and Rabczuk and Zi [14], Rabczuk and Belytschko [15] and weight-function based enrichment as in Duflot and Nguyen-Dang [16] and more recently Barbieri et al. 17], are now commonly accepted versatile tools to handle arbitrary crack paths and dislocations Gracie et al. [18].

The idea of this paper is to show how, for three-dimensional crack problems, molecular dynamics can be coupled to continuum methods in order to obtain a technique which focuses the computational effort where it is most required to capture the relevant Physics of crack and dislocation initiation and growth. This affords the modelling and simulation of relatively long cracks, by restricting the MD model to a small zone around the crack front. Ultimately, this approach also makes it possible to adaptively refine or coarse-grain the atomistic region where appropriate [19].

The methodology adopted in this work relies on two ingredients. The first is partition of unity enrichment for discontinuities in the continuum region. The second is the bridging domain method (BDM), inspired by the Arlequin method of Ben Dhia H [3] and by the coupling methods of S.P. Xiao [2], Mei Xu [20] and Gracie and Belytschko [21]. These energy-coupling methods enforce a weak coupling between the models in the two adjacent domains. It was shown in Mei Xu [20] that spurious wave reflection is limited when using this method.

The advantage of the coupled XFEM-BDM method over most concurrent multiscale methods such as the standard BDM or the quasi-continuum method of Tadmor et al. [1], Shenoy et al. 22] and Miller and Tadmor [23] is that the XFEM-BDM requires the atomistic region to be present only around the crack front, and not along the whole length of the crack surface to represent the discontinuity across the crack faces. The enrichment of the finite elements in the 
handshake and continuum parts of the domain allows cracks to be smoothly represented from the continuum model to the atomistic model, through the transition region.

The BDM and the XFEM were first coupled, in the context of two-dimensional problems by P. Aubertin [24, 25], R. Gracie [26]. Yet, crack problems are inherently three dimensional, where the XFEM-Bridging Domain method is not well tested. The three dimensional problem introduces a range of difficulties, demanding the development of new algorithms. These problems include locating the crack and dislocations within the MD domain and other implementation difficulties discussed briefly in this paper.

This paper is organized as follows. First, the governing equations are stated. Then the crack modelling in continuum domains using the extended finite element approach is discussed briefly. Next the coupling method and coupling force calculations are detailed, before implementation and numerical examples are presented.

\section{Model Description and Governing Equations}

\subsection{Definitions}

Consider a domain $\Omega$ where an existing crack with surface $\partial \Omega_{c}$ is to be simulated. We introduced an overlapping decomposition of the domain $\Omega$ into domain $\Omega^{\mathrm{fs}}$ where the material is modelled by $\mathrm{MD}$, and domain $\Omega^{\mathrm{cs}}$ where the system is described by a continuum formulation relying on the Cauchy-Born rule as constitutive model 1 . The shape of $\Omega^{\mathrm{fs}}$ is introduced a priori in this study (this restriction will be discussed in the conclusion), and is such that $\Omega^{\mathrm{fs}}$ contains the crack front of the macroscopic crack. The overlap region $\Omega^{\mathrm{B}}=\Omega^{\mathrm{cs}} \cap \Omega^{\mathrm{fs}}$ is the so-called bridging domain. The outer boundary of $\Omega^{\mathrm{cs}}$ is denoted by $\partial \Omega^{\mathrm{cs}}$ with $\partial \Omega^{\mathrm{cs}}=\partial \Omega_{t}^{\mathrm{cs}} \cup \partial \Omega_{u}^{\mathrm{cs}} \cup \partial \Omega_{c}^{\mathrm{cs}}$ and $\partial \Omega_{t}^{\mathrm{cs}} \cap \partial \Omega_{u}^{\mathrm{cs}}=\emptyset, \partial \Omega_{c}^{\mathrm{cs}} \cap \partial \Omega_{u}^{\mathrm{cs}}=\emptyset, \partial \Omega_{t}^{\mathrm{cs}} \cap \partial \Omega_{c}^{\mathrm{cs}}=\emptyset$; subscripts $u, t$ and $c$ indicate 'displacement', 'traction-' and 'crack-', respectively. Notations are summarized in Fig. 1. This molecular treatment of the crack front zone enables the description of voids, dislocations and cracks which are known to strongly influence the initiation and propagation of cracks, see, e.g. Lange [27] and Ravi-Chandar and Knauss [28].

In this study, a base crystalline material with a given lattice structure is assumed everywhere. The fine scale region $\Omega^{\mathrm{fs}} \backslash \Omega^{\mathrm{B}}$ is composed of the atoms of this base lattice structure and the

\footnotetext{
${ }^{1}$ In this paper, the superscripts fs and cs denote the fine scale and coarse scale, respectively.
} 


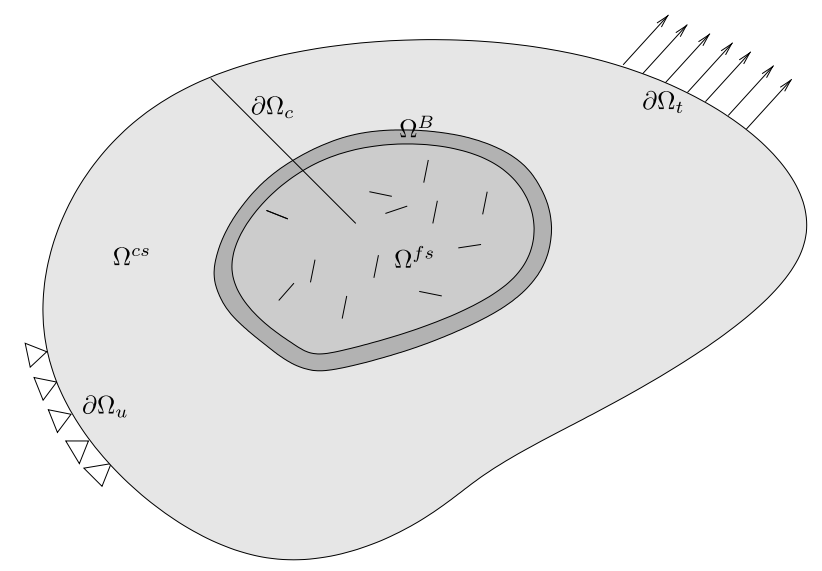

Figure 1: The fine and coarse scale overlapping domains.

finite elements in this region are deactivated. This is shown in Fig. 2, In the continuum region, $\Omega^{\mathrm{cs}} \backslash \Omega^{\mathrm{B}}$, the continuum model is activated where the material behaviour is approximated by the Cauchy-Born rule (Tadmor et al. [1]). The idea of the Cauchy-Born rule is to compute the material properties and stress from the same potential used in the fine scale domain $\Omega^{\mathrm{fs}}$, here the Lennard-Jones potential (LJP). In other words at every point of the continuum model, we have a very small atomistic model with the same lattice structure and force fields. The interested reader can refer to studies by Aghaei et al. [29] and Pozrikidis [30] on the applicability and stability of the Cauchy-Born method.

In the bridging (or handshake/blending) domain $\Omega^{\mathrm{B}}=\Omega^{\mathrm{cs}} \cap \Omega^{\mathrm{fs}}$, the continuum and atomistic descriptions cohabit. In the bridging domain, the continuum energy and the atomistic energy are blended through a weighting by functions summing to unity. One criterion which should be fulfilled is that the sum of the continuum and the atomistic energy is unchanged by the coupling method. From a kinematic point of view, in this sub-domain, the continuum model is coupled to the atomistic model by enforcing compatibility of the displacements of the atoms and that of the nodes.

\subsection{The Displacement Approximation}

Let the reference and the current configurations of the domain be denoted by $\Omega_{0}$ and $\Omega$, respectively. The number of atoms in sub-domain $\Omega^{\mathrm{fs}}$ is denoted by $n^{\mathrm{fs}}$. Let the number of ghost or pad atoms be denoted by $n^{G}$. Ghost atoms are those atoms located in the continuum region $\Omega^{\mathrm{cs}}$ that are within the cut-off radius of atoms in the atomistic region $\Omega^{\mathrm{fs}}$. The positions of ghost 


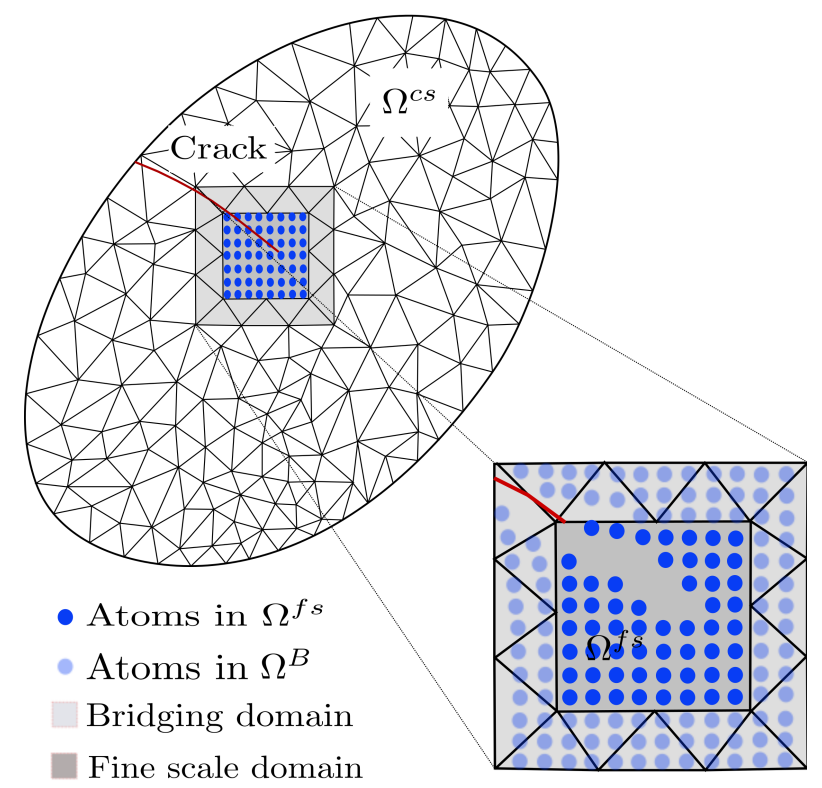

Figure 2: The solution domain including the crack and the bridging domain.

atoms are interpolated from the continuum solution. The material coordinates of a point in $\Omega_{0}^{\mathrm{cs}}$ are denoted by $\mathbf{X}$ and the spatial coordinates by $\mathbf{x}$. The location of atom $\alpha$ in the reference and spacial configurations will be denoted by $\mathbf{X}_{\alpha}$ and $\mathbf{x}_{\alpha}$ respectively.

The atomistic evolution of the material in time and space is approximated by now wonted molecular dynamics formulations, details of which are given in a body of literature, such as Haile [31], Rapaport [32]. In the continuum sub-domain, the displacement field is approximated by the extended finite element method (XFEM). In this approximation, the displacement field is decomposed into a continuous part and a discontinuous part as in Belytschko and Black [8]:

$$
\boldsymbol{u}^{h}(\boldsymbol{X})=\underbrace{\sum_{I \in \mathcal{N}} N_{I}(\boldsymbol{X}) \boldsymbol{u}_{I}}_{\mathbf{u}^{\text {cont }}}+\underbrace{\sum_{I \in \mathcal{N}_{b}} N_{I}(\boldsymbol{X}) H\left(f_{I}(\boldsymbol{X})\right) \boldsymbol{a}_{I}}_{\mathbf{u}^{\text {discont }}}
$$

where $\mathcal{N}$ is the set of all nodes in the domain and $\mathcal{N}_{b}$ is the set of nodes that belong to all elements which are completely cut by the crack. The nodal unknowns $\boldsymbol{u}_{I}$ and $\boldsymbol{a}_{I}$ are standard and enriched degrees of freedom respectively and $H$ is the discontinuous enrichment (Heaviside) function: 


$$
H(f(\boldsymbol{X}))=\left\{\begin{array}{lll}
1 & \text { if } & f(\boldsymbol{X})>0 \\
0 & \text { if } & f(\boldsymbol{X})<0
\end{array}\right.
$$

with

$$
f(\boldsymbol{X})=\operatorname{sign}\left[\boldsymbol{n} \cdot\left(\boldsymbol{X}_{I}-\boldsymbol{X}\right)\right] \min _{\mathbf{X}_{\mathbf{I}} \in \partial \Omega_{c}}\left\|\boldsymbol{X}_{I}-\boldsymbol{X}\right\|
$$

where $\boldsymbol{n}$ is the outward normal to the crack surface. In this study since the crack front is modelled by the atomistic region, no crack-front enrichment is needed.

\subsection{The Handshake Region}

Consider an FEM discretization of the entire domain $\Omega_{0}$. Let the set of nodes and the set of elements of this mesh be denoted by $\mathcal{N}$ and $\mathcal{E}$, respectively. As described above, we will weigh the contributions of the atomistic and continuum models in the bridging domain $\Omega^{\mathrm{B}}$ relative to the total energy of the system. To accomplish this we define a weight function which is unity outside the atomistic domain, zero inside the atomistic domain, and smooth in the blending region. A range of admissible such weight functions $w$ writes:

$$
w(\boldsymbol{X})=\left\{\begin{array}{cc}
1 & \forall \mathbf{X} \in \Omega^{\mathrm{cs}} \backslash \Omega^{\mathrm{fs}} \\
{[0,1]} & \forall \mathbf{X} \in \Omega^{\mathrm{B}} \\
0 & \forall \mathbf{X} \in \Omega^{\mathrm{fs}} \backslash \Omega^{\mathrm{cs}}
\end{array}\right.
$$

In order to define $w$ at any point $\mathbf{X}$ we compute a normalized distance function by:

$$
w(\boldsymbol{X})=\frac{l(\boldsymbol{X})}{l_{0}}
$$

where $l(\boldsymbol{X})$ is the orthogonal projection of $\boldsymbol{X}$ on the interior boundary of the continuum scale sub-domain $\Omega^{\mathrm{cs}}$ and $l_{0}$ is the length of this orthogonal projection to the boundary of the atomistic region $\Omega^{\text {fs }}$. Fig. 3 gives a schematic description of these definitions.

The weighting parameter is constant and defined according to the material coordinate. This means that the weighting parameter is computed once in the beginning of the simulation and 


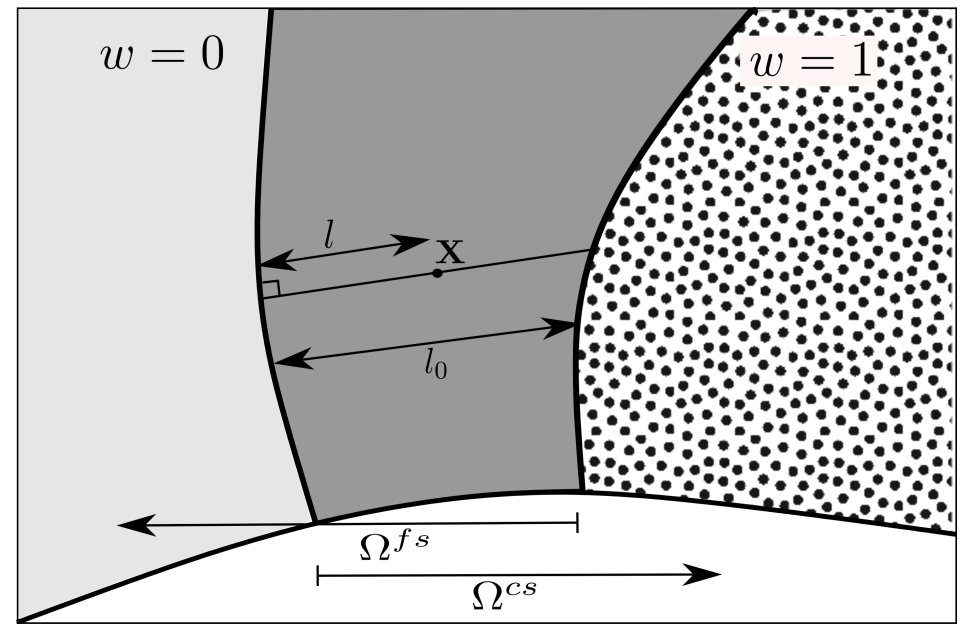

Figure 3: Calculation of the weighting function in the bridging domain in two dimensions

with the unreformed configuration. In practice, we just locate the continuum nodes with $w=0$ and $w=1$ and we interpolate the weighting of other points such as atoms and integration points from the finite element nodes. Thus, the weighting function defines the continuum nodes and elements and active atoms during solution.

\subsection{Coupling Method}

The coupling method for BDM is comprehensively described in the work of S.P. Xiao [2], Mei Xu [20], G. Anciaux [33], Guidault PA [34] therefore we only briefly explain the governing equations. The governing equations are derived from the Lagrangian of the systems, L, which is the sum of the Lagrangians of each subdomain:

$$
\mathrm{L}=\mathrm{L}^{\mathrm{cs}}+\mathrm{L}^{\mathrm{fs}}+\mathrm{L}^{\mathrm{B}}
$$

where $\mathrm{L}^{\mathrm{fs}}$ and $\mathrm{L}^{\mathrm{cs}}$ are the Lagrangians of the atomistic and continuum sub-domains, respectively and $L^{B}$ is the energy contribution from the enforcement of compatibility between the atomistic and the continuum displacements in the bridging domain $\Omega^{\mathrm{B}}$.

In the following, lower case indices indicate coordinate directions, e.g. $i=1,2,3$ and upper case indices indicate finite element nodes and atoms, e.g. $I$.

The semi-discrete equations of motion can be obtained from the Lagrange equations of motion: 


$$
\begin{aligned}
& \frac{d}{d t}\left(\frac{\partial \mathrm{L}}{\partial \dot{u}_{I i}}\right)-\frac{\partial \mathrm{L}}{\partial u_{I i}}=0 \\
& \frac{d}{d t}\left(\frac{\partial \mathrm{L}}{\partial \dot{d}_{\alpha}^{\mathrm{fs}}}\right)-\frac{\partial \mathrm{L}}{\partial d_{\alpha}^{\mathrm{fs}}}=0
\end{aligned}
$$

where $\dot{u}_{I i}$ and $\dot{d}_{\alpha}^{\text {fs }}$ are the time derivatives of $u_{I i}$ and $d_{\alpha}^{\text {fs }}$ respectively.

For the coarse scale, continuum, sub-domain, the Lagrangian is:

$$
\mathrm{L}^{\mathrm{cs}}=\frac{1}{2} \int_{\Omega_{0}^{\mathrm{cs}}}(1-w(\mathbf{X})) \rho_{0} \dot{\mathbf{u}}^{h} \cdot \dot{\mathbf{u}}^{h} d \Omega_{0}^{\mathrm{cs}}-\int_{\Omega_{0}^{\mathrm{cs}}}(1-w(\mathbf{X})) W(\mathbf{F}) d \Omega_{0}^{\mathrm{cs}}-W^{\mathrm{ext}}
$$

where $\mathbf{F}=F_{i j}$ is the deformation gradient, $\rho_{0}$ is the initial density, $W^{\text {ext }}$ is the work of the external loads and $W(\mathbf{F})$ is the strain energy density of the continuum which is determined from the Cauchy-Born rule [35].

For the fine scale, atomistic, subdomain, the Lagrangian is:

$$
L^{\mathrm{fs}}=\frac{1}{2} \sum_{\alpha=1}^{n^{\mathrm{fs}}} w\left(\mathbf{X}_{\alpha}\right) m_{\alpha}^{\mathrm{fs}} \dot{\mathbf{d}}_{\alpha}^{\mathrm{fs}} \cdot \dot{\mathbf{d}}_{\alpha}^{\mathrm{fs}}-\sum_{\alpha=1}^{n^{\mathrm{fs}}} \sum_{\beta>\alpha}^{n^{\mathrm{fs}}+n^{G}} \frac{1}{2}\left(w\left(\mathbf{X}_{\alpha}\right)+w\left(\mathbf{X}_{\beta}\right)\right) V\left(r_{\alpha \beta}\right)
$$

where $m_{\alpha}^{\text {fs }}$ is the mass of atom $\alpha, r_{\alpha \beta}$ is the distance between the two atoms $\alpha$ and $\beta$ and $V\left(r_{\alpha \beta}\right)$ is the two body potential relating the atoms. We assume a cut-off radius outside of which atomic forces are zero.

The constraints from the coupling region write

$$
g_{\alpha i}(t)=u_{i}^{\mathrm{cs}}\left(\mathbf{X}_{\alpha}, t\right)-d_{i \alpha}^{\mathrm{fs}}(t)
$$

Applying the above constraint to the Lagrangian is done here by using Lagrange multipliers $\lambda_{\alpha}:$

$$
\mathrm{L}^{B}=\sum_{\alpha \in \Omega_{0}^{B}} \lambda_{\alpha} \cdot g_{\alpha}
$$

It is notable that the Lagrange multipliers are assigned to the discrete positions of atoms in the bridging domain. Finally, we obtain the following semi-discrete equations of motion: 


$$
\begin{aligned}
& \forall I \in \mathcal{N}^{\mathrm{cs}} \forall i \in\{1,2,3\}: M_{I J} \ddot{u}_{J i}=f_{I i}^{\mathrm{ext}}-f_{I i}^{i n t}+f_{I i}^{\lambda c s}, \\
& \forall \alpha \in \llbracket 1, \ldots, n^{\mathrm{fs}} \rrbracket, \forall i \in\{1,2,3\}: m_{\alpha}^{\mathrm{fs}} \ddot{d}_{\alpha i}^{\mathrm{fs}}=f_{\alpha i}^{\mathrm{fs}}+f_{\alpha i}^{\lambda f s},
\end{aligned}
$$

where $\ddot{u}_{J}$ and $\ddot{d}_{\alpha}^{A}$ are the accelerations of node $J$ and atom $\alpha$, respectively. Also, the mass matrix is computed by:

$$
\forall I, J \in \mathcal{N}^{\mathrm{cs}}: M_{I J}=\int_{\Omega_{0}^{\mathrm{cs}}}(1-w) \rho_{0} N_{I} N_{J} d \Omega_{0}^{\mathrm{cs}}
$$

The internal forces in the continuum domain write:

$$
\forall I \in \mathcal{N}^{\mathrm{cs}}, \forall i \in\{1,2,3\}: f_{I i}^{i n t}=\int_{\Omega_{0}^{\mathrm{cs}}}(1-w) P_{i j} \frac{\partial N_{I}}{\partial X_{j}} d \Omega_{0}^{\mathrm{cs}},
$$

where $\mathbf{P}=P_{i j}$ is the nominal stress. From the Cauchy-Born rule it writes $\mathbf{P}(\mathbf{F})=\frac{\partial W(\mathbf{F})}{\partial \mathbf{F}}$. The forces on each atom are determined from the interatomic potential $V$ as

$$
\forall \alpha \in \llbracket 1 \ldots n^{\mathrm{fs}} \rrbracket, \forall i \in\{1,2,3\}: f_{\alpha i}^{\mathrm{fs}}=-\sum_{\beta} \frac{1}{2}\left(w\left(X_{\alpha}\right)+w\left(X_{\beta}\right)\right) \frac{\partial V\left(r_{\alpha \beta}\right)}{\partial d_{i \beta}^{\mathrm{fs}}}
$$

where $\beta$ ranges over all atoms within the cutoff radius of atom $\alpha$. The forces $f^{\lambda c s}$ in the continuum region and $f^{\lambda f s}$ in the atomistic region, due to the coupling are given by:

$$
\forall I \in \mathcal{N}^{\mathrm{cs}}, \forall i \in\{1,2,3\}: f_{I i}^{\lambda c s}=\sum_{\alpha \in \Omega_{0}^{B}} \lambda_{\alpha i} N_{I}\left(X_{\alpha}\right)
$$

and

$$
\forall \alpha \in \llbracket 1 \ldots n^{\mathrm{fs}} \rrbracket, \forall i \in\{1,2,3\}: f_{\alpha i}^{\lambda f s}=-\lambda_{\alpha i} .
$$

Remark 1 To compute the Lagrange multiplier unknowns we use the method described by T. Belytschko [36].

Remark 2 The mass matrix in the continuum region is diagonalized according to the masslumping scheme for XFEM which was proposed by Menouillard et al. [37]. 


\section{Some Implementation Details}

\subsection{Molecular Dynamics}

The fact that continuum and atomistic (discrete) domains are coupled creates certain difficulties in the implementation due to the different nature of the information available in both domains. For the atomistic part a code named WARP from Plimpton [38] is adopted here. WARP is a parallel molecular dynamics simulation code and antecedent of LAMMPS Molecular Dynamics Simulator of Plimpton [39] for modelling stress and strain in materials using the embedded atom method (EAM), Modified embedded atom method (MEAM) and Lennard-Jones (LJ) potentials. It is written in Fortran 90 and performs message-passing via MPI calls. WARP already has a very efficient neighbour search and force computation algorithms which are essential in three dimensional simulations.

The WARP code is modified and merged into an extended finite element library called PERMIX (based on Fortran 2003 standard). The potentials in WARP had to be also modified to account for weighting of the atoms in the bridging domain. Also, to implement the CauchyBorn rule, a very small atomistic part is defined at the integration point level of the coarse scale which handles stress and stiffness calculation. The usage of the WARP code over the current version of LAMMPS is attributed to its ease of integration into our in-house XFEM library since both programs are written in the Fortran language and the WARP code was easier to modify. However, using the current version of LAMMPS will be considered in the future to use its many capabilities over WARP.

In our implementation we create Lagrange multiplier mesh with the same fineness as the atomistic spacing. The size of the coupling region is based on the finite element mesh and it is one element thick. In this study, we did not notice any instability due to spurious wave reflections.

\subsection{Three Dimensional XFEM}

XFEM was used in this study to represent the crack surface during the simulation. In general, XFEM possesses many advantages over finite elements for crack simulation such as meshing problems. However, in comparison to pure Finite Elements and especially in three dimensions, the XFEM formulation introduces some difficulties in the implementation. Some of the 
difficulties include initial crack surface input, integration and post-processing.

To create the initial crack surface, we directly enter the level sets associated with every cracked node and element. Theses level sets are created by the ABAQUS software package

[40]. Therefore the implementation will be very general and can be applied to various problems. There are standard methods to integrate fields in XFEM elements [41] where we tetrahedralize the XFEM elements and create the integration points within every tetrahedron. We use same tetrahedrons for post-processing purposes where we project the fields to the nodes of the tetrahedrons.

\section{Numerical example}

Consider a three dimensional single crystal with a face centred cubic (FCC) lattice which has dimensions $1,000 \times 1,000 \times 150 \AA^{3}$. In this example a straight crack of length $500 \AA$ is assumed present in the domain across the whole thickness. Along the bottom boundary of the continuum domain, all degrees of freedom are fixed to zero. The continuum model consists of 44,890 hexagonal elements and 152,592 degrees of freedom. The element size is constant over the domain, about $15 \AA$. An atomistic domain of size $365 \times 365 \times 150 \AA^{3}$ is placed centred around the crack front. Fig. 4 shows a schematic configuration of the system.

Since part of the crack falls within the atomistic domain, the crack must be modelled in the atomistic region as well as in the continuum region. We do not follow the generally adopted method of removing rows of atoms along the crack, as this is somewhat arbitrary and introduces extra parameters in the formulation. Instead, we modify the neighbour list of the atoms to prevent force transmission across the crack faces. This is done as follows. The initial crack position allows us to define two groups of atoms: group 1 is composed of atoms above the crack, and group 2 of atoms located below the crack. During the neighbor search, we forbid atoms of group 1 to become neighbors of atoms of group 2, and vice versa. As cracks propagate, or for more complex crack geometries, a criterion similar to the "visibility criterion" used in meshless method:2 could be used. The method that we used here to introduce a crack can be used generally for other potentials such as EAM and MEAM since it is based on the neighbour lists and not the potential itself. This method will produce a crack which is consistent with a

\footnotetext{
${ }^{2}$ see the recent review on (enriched) meshless methods by Nguyen et al. [42]
} 
sharp XFEM crack.

The use of XFEM for the current example seems to be unnecessary since the initial crack surface is straight and the crack is not propagated into the continuum field. Nevertheless, our implementation is usable for a very general case of complicated geometry and crack surface. Also, the extension of this method to a simulation with coarse graining will be straight forward and the advantages of XFEM over Finite Elements are trivial in that case.

The atomistic domain is a three dimensional lattice from an FCC crystal with lattice constant $3.645 \AA$ extended in the $\left[\begin{array}{lll}1 & 0 & 0\end{array}\right]$ crystal direction. Atomic interactions are modelled by the Lennard-Jones potential with parameters $\sigma=2.29621 \AA$ A $\epsilon=0.467 \mathrm{eV}$, and a cut-off radius of $4.0 \AA$; the mass of all atoms is taken as $65 \frac{\mathrm{g}}{\mathrm{mol}}$. In this study, we have not taken the temperature into account, since the focus was on the coupling of (extended) finite element method with Molecular Dynamics. However, in our development, we can easily control the temperature in the atomistic domain with Nose-Hoover thermostat method by S. [43]. To be able to model a realistic three dimensional problem, where periodicity is usually not available, we have not used any periodic boundary in the system. Therefore, the only type of boundary condition employed in this example in the atomistic region is that coming from the coupling region.

Before the actual coupled simulation starts, we have minimized energy in the pure atomistic part to relax the system. We have used the the conjugate gradient (CG) algorithm [44] to minimize the energy of the system. At each iteration the force gradient is combined with the previous iteration information to compute a new search direction perpendicular (conjugate) to the previous search direction.

The coupling of the continuum and atomistic part is performed within a cubic box with of dimensions $310 \times 310 \times 150 \AA^{3}$. The elements which are cut by this box are the bridging elements and the atoms which are located inside bridging elements are the bridging atoms. Consequently, the coupling region is one element wide. With this configuration the model has 1,368,575 active atoms, 231,890 bridging atoms and 308,067 ghost atoms.

The driving force for the system is introduced through a velocity boundary condition on the top face of the continuum region. A velocity of $0.1 \AA /$ pico seconds is set on all the nodes belonging to the top boundary of the continuum domain, at every time step. The time step is 0.003 picoseconds and the total simulation time is 1200 picoseconds. The magnitude of the time 


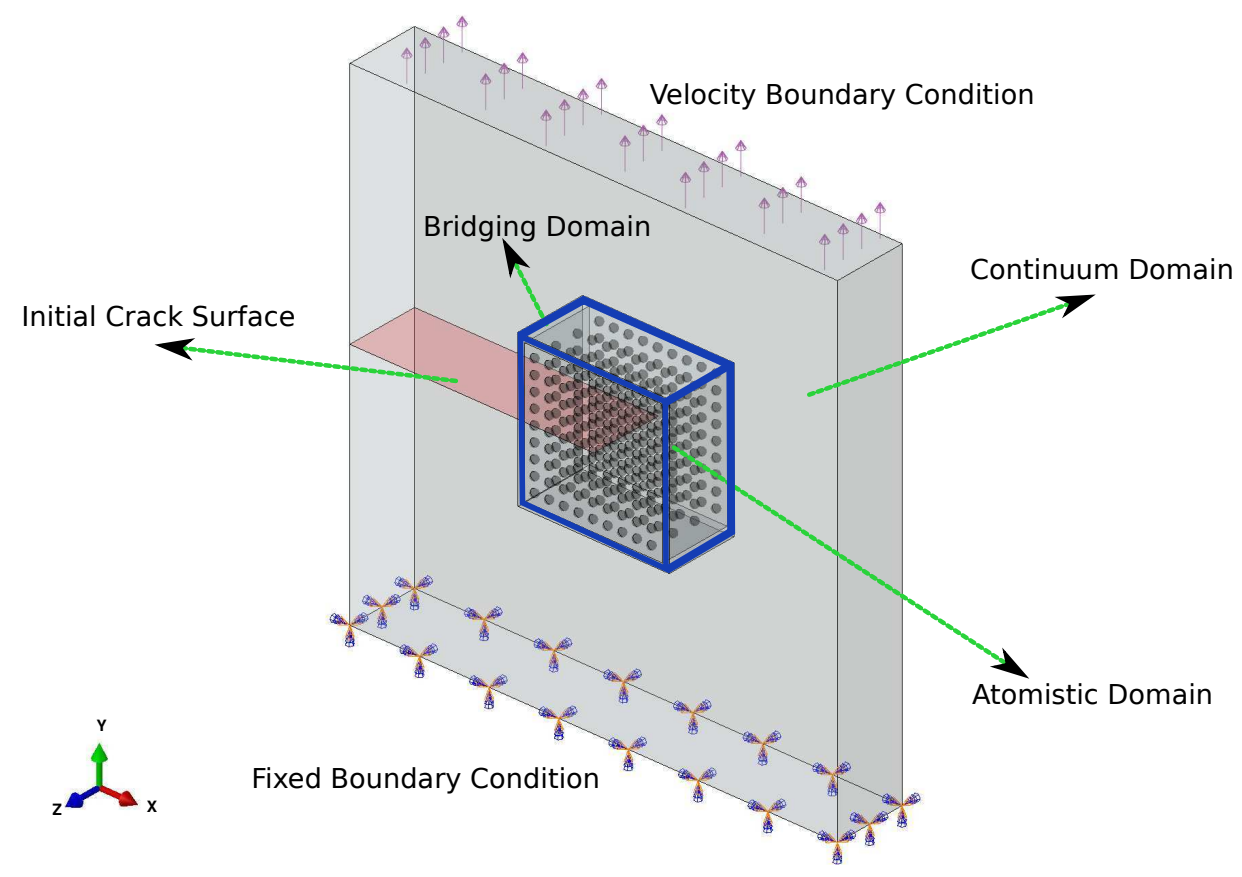

Figure 4: Schematic view of the example, bridging domain, atomistic domain and boundary conditions for a mode I crack.

step is chosen according to the time step suitable for the pure atomistic simulation; therefore not multiple time-stepping is employed here. Fig. [5] shows the initial configuration of the body and the weighting of the atoms and nodes.

Fig. 7 shows the atoms with higher centro-symmetry value, at different time steps. For the current system the centro-symmetry parameter is a powerful measure of the local lattice disorder around an atom and may be used to characterize and visualize whether the atom belongs to a perfect lattice, a defect (e.g. a stacking fault or a dislocation), or a surface [45]. The centro-symmetry indicator $C S$ is computed as explained in Kelchner et al. [46]:

$$
C S=\sum_{i=1}^{\frac{N}{2}}\left|\mathbf{R}_{i}+\mathbf{R}_{i+\frac{N}{2}}\right|^{2},
$$

where $N$ is the number of nearest neighbours for each atom in the underlying lattice of atoms. For example here $N=12$ for the FCC lattice. $\mathbf{R}_{i}$ and $\mathbf{R}_{i+\frac{N}{2}}$ are vectors from the atom of interest to a particular pair of nearest neighbours. The value in the sum is computed for each atom, and the $N / 2$ smallest quantities are used. For an atom on a lattice site, surrounded by atoms on a perfect lattice, the centro-symmetry parameter will be 0 . It will be also near 0 for small thermal perturbations of a perfect lattice. 


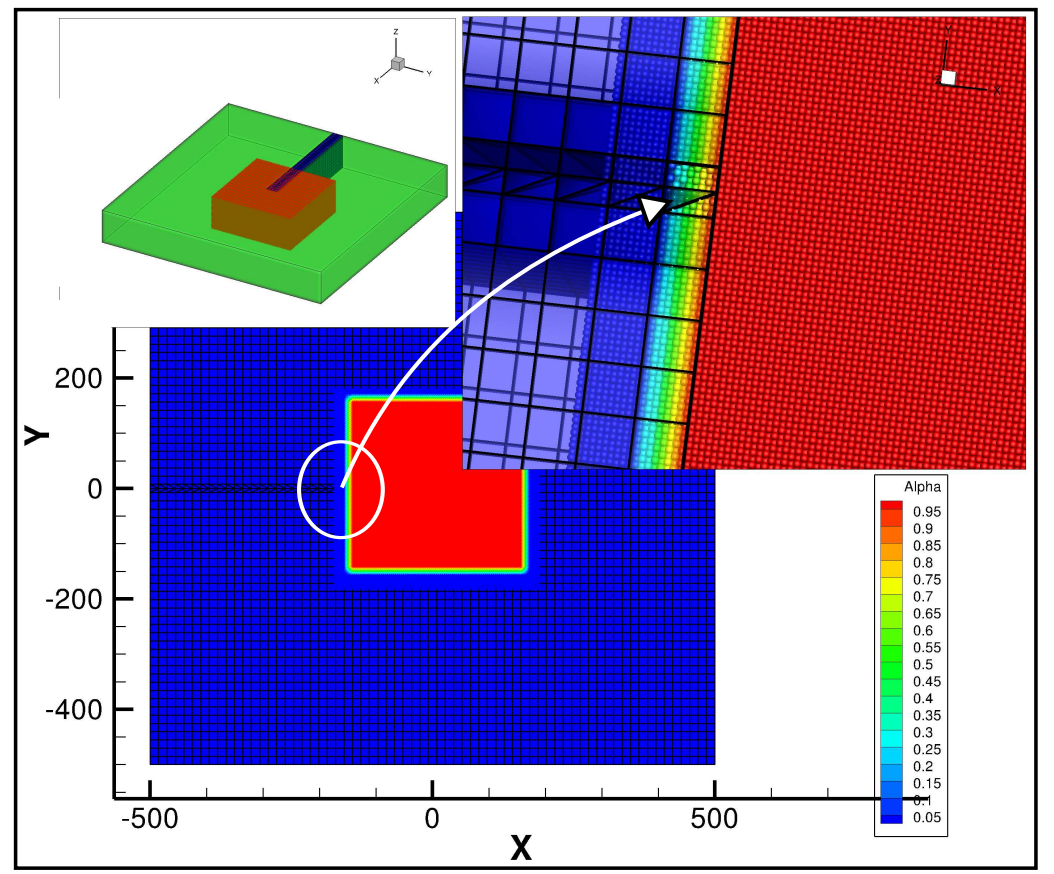

Figure 5: Initial configuration of the system and the contour of the weighting values at the atoms and nodes. 
From Fig. 7 we realize that although, from a continuum point of view, the boundary conditions suggest an almost mode I loading of the crack, which should consequently propagate normally to the loading direction, we do not observe this behaviour here.

In the atomistic region, the nucleation and propagation of dislocations (in blue in Figs. 6(a) 6(b) and 6(c) , and the creation of free surfaces (in green in the same figures) are strongly influenced by surface effects. These effects lead to anisotropy and hence to deviations from behaviours expected from isotropic continuum theory. Also, in this example we have not applied boundary conditions which suggest pure Mode I failure from continuum theory since the bottom of the system is fully clamped. Moreover, the anisotropic behaviour in the pure atomistic simulation of single FCC crystals have been studied extensively such as in [47, 48]. Notice in Fig. 7(c) the triangular shape of the propagating crack and the dislocations nucleating from the boundaries of the domain.

In Fig. $7(\mathrm{~d})$ it is seen that the dislocations have propagated and have just reached the coupling region. At this point, the model is not valid any more since the dislocations should pass into the continuum region or the atomistic region should be adaptively enlarged to handle the dislocation propagation. There are two methods which may be used to pass the dislocations to the continuum region. First is the one suggested by Gracie and Belytschko [19] which describes the dislocations in terms of XFEM enrichment. The second method is offered by Xiong et al. [49] where a new continuum field formulation of balance laws with relevant atomistic information (the arrangements and interactions of atoms) considered to represent dislocations by the continuum region.

The choice of adaptively enlarging the atomistic region as opposed to using methods to handle dislocations in the continuum region is problem dependent. For example, in problems where the size of the region with dislocation nucleation and growth are confined, we can effectively use the former method. The latter method seems more useful for the current problem since eventually a big portion of the system should be handled by pure atomistic formulation.

Fig. 8 shows the stress contours at four different time steps which are the same time steps as in Fig. 7. In this figure, the cross section of the specimen is shown for better visualization of stress fields around the crack surface. The atomistic stress computed here is the virial stress tensor. The symmetric virial stress tensor is computed for every atom and for pair potentials 




(a)



(b)

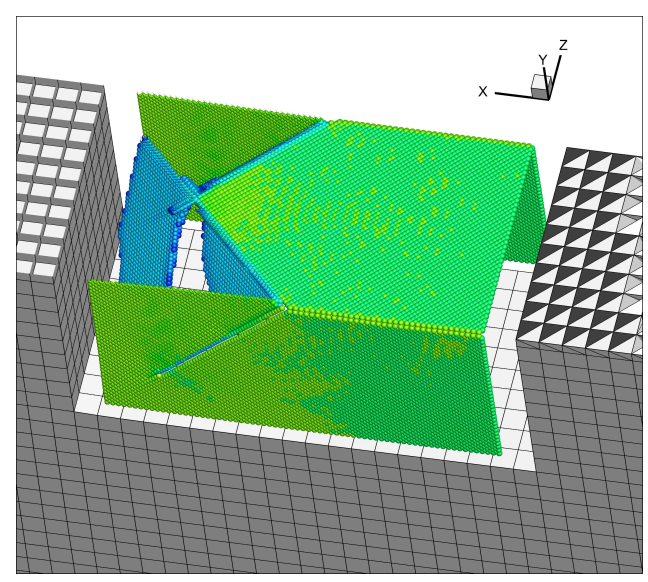

(c)

Figure 6: Pictorial representation of the propagating crack (free surfaces represented in green) and the initiated dislocations (represented in blue). 


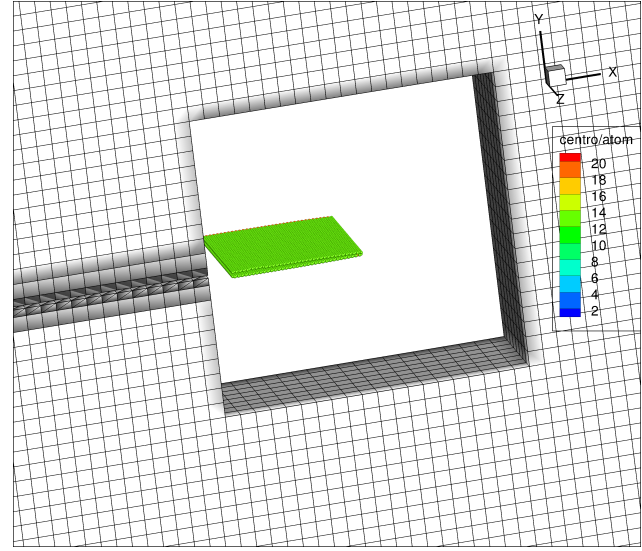

(a)

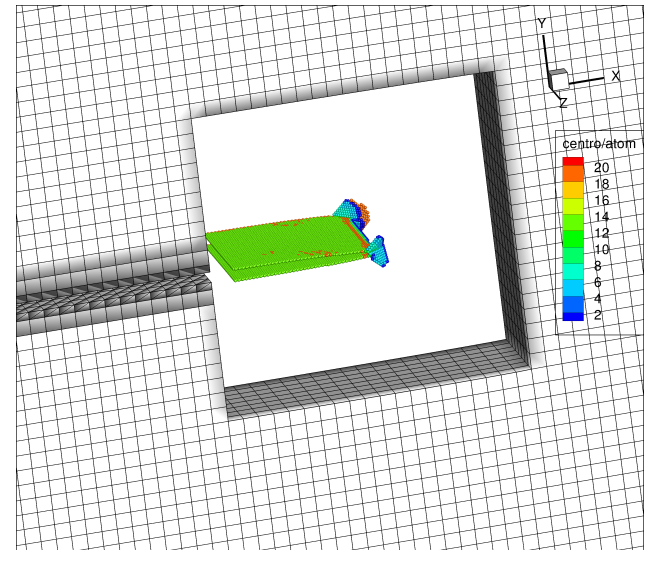

(c)

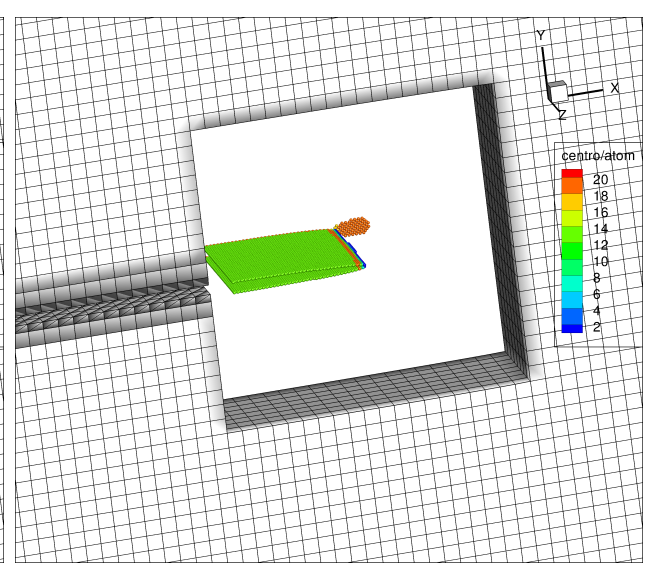

(b)

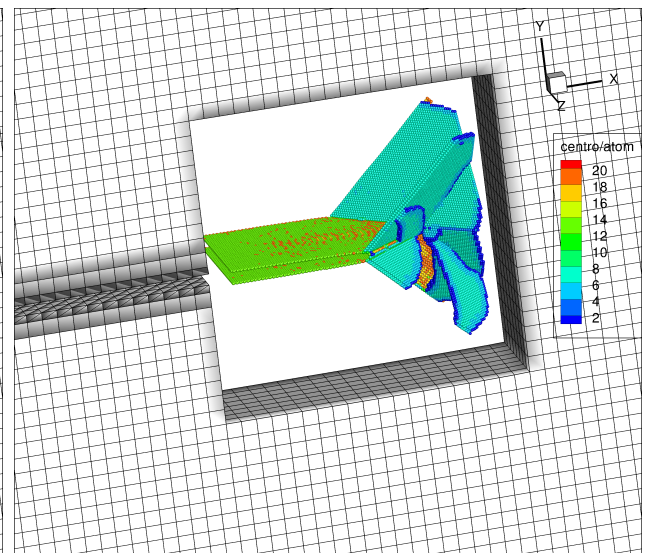

(d)

Figure 7: The propagation of the crack front and dislocations shown with atoms with high centro-symmetry value in different time steps (a) step 10000, (b) step 33000, (c) step 34000, (d) step 35800 . 
such as the one used here is defined in Robert [50] and Subramaniyan and Sun [51]:

$$
\sigma_{i j}^{V}=\frac{1}{V} \sum_{\alpha}\left[\frac{1}{2} \sum_{\beta=1}^{N}\left(R_{i}^{\beta}-R_{i}^{\alpha}\right) F_{j}^{\alpha \beta}-m^{\alpha} v_{i}^{\alpha} v_{j}^{\alpha}\right]
$$

where $(i, j)$ range over the spatial directions, $x, y, z . \beta \in \llbracket 1, \ldots, N \rrbracket$ ranges over the $N$ neighbors

of atom $\alpha, R_{i}^{\alpha}$ is the coordinate of atom $\alpha$ in the $i$ direction, $F_{j}^{\alpha \beta}$ is the force on atom $\alpha$ from atom $\beta$ along the $j$ direction, $V$ is the total volume, $m^{\alpha}$ is the mass of atom $\alpha$ and $v^{\alpha}$ is the velocity of atom $\alpha$. The definition of virial stress involves the instantaneous velocities only due to thermal fluctuations. While Eq. 21 is temperature dependent, we have neglected these effects. This assumption will not in general affect the qualitative results discussed here since the first term in Eq. 21 dominates the second term and will not influence the stress concentration regions. Note that to obtain the equivalent continuum Cauchy stress, the virial stress from the molecular dynamics (MD) simulations has to be averaged over time and space, as explained in Bühler [52]. In Fig. 8(a) and (b) a stress concentration is visible, that is initially confined at the crack front; subsequently when propagation occurs, the stress waves are emitted from the crack tip. From Fig. 8(b) and (c) the surface effects of the third dimension are also evident. From this figure we also notice the stress distribution around the dislocations and the crack. Such complex mechanisms of crack and dislocations could not be predicted by any classical continuum description of motion.

\section{Conclusion}

We presented a coupling method for bridging a three dimensional extended finite element treatment of cracks and molecular dynamics. This method is based on an overlapping domaindecomposition scheme where the displacement compatibility conditions in the overlapping subdomain are enforced by Lagrange multipliers. We showed how the method can be successfully used to simulate the propagation of a crack in quasi-mode I, where an atomistic domain is placed on top of the three dimensional extended finite element domain, around the crack front. We also computed the centro-symmetry parameter and virial stress tensor in the atomistic region. We have observed that our three dimensional coupled method is capable of representing the crack and dislocation propagation for much fewer degrees of freedom than a direct numerical 


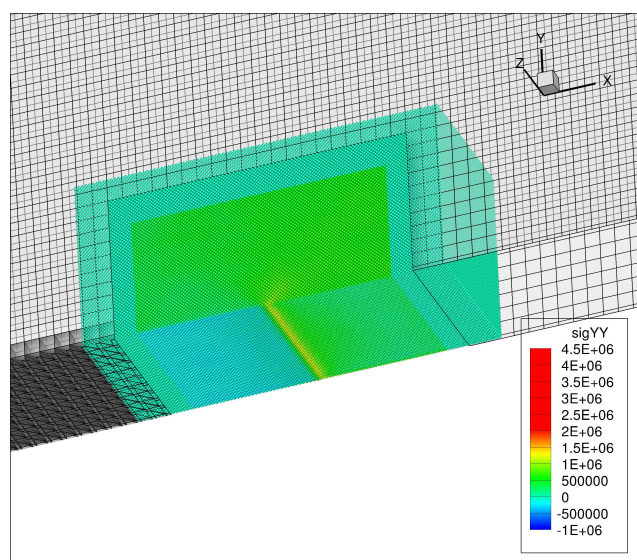

(a)

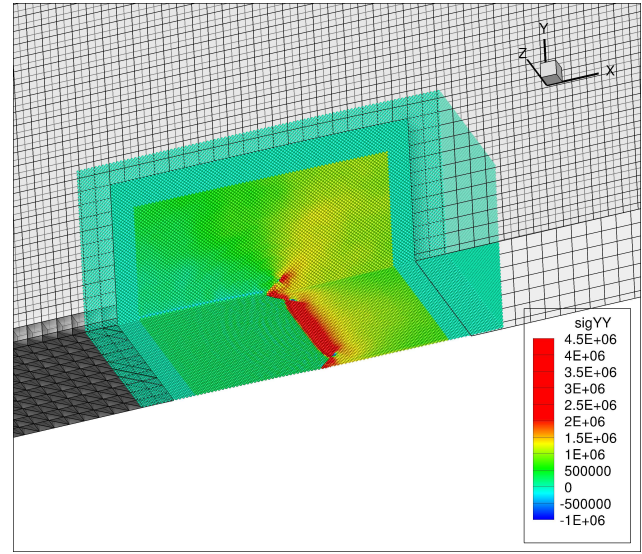

(c)

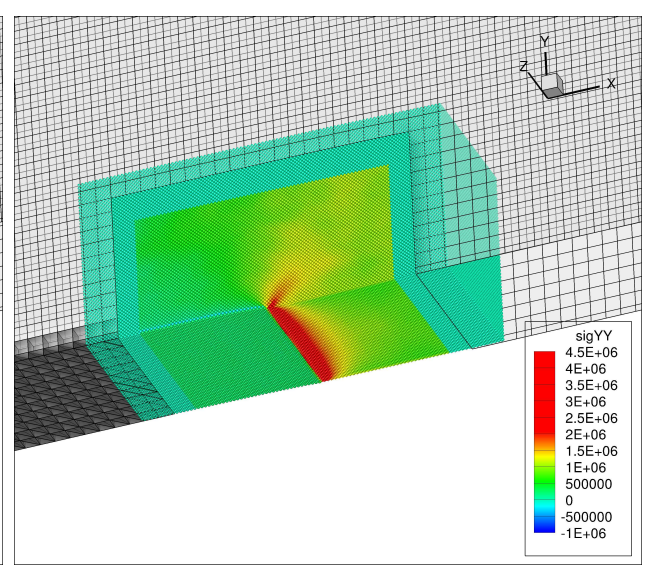

(b)

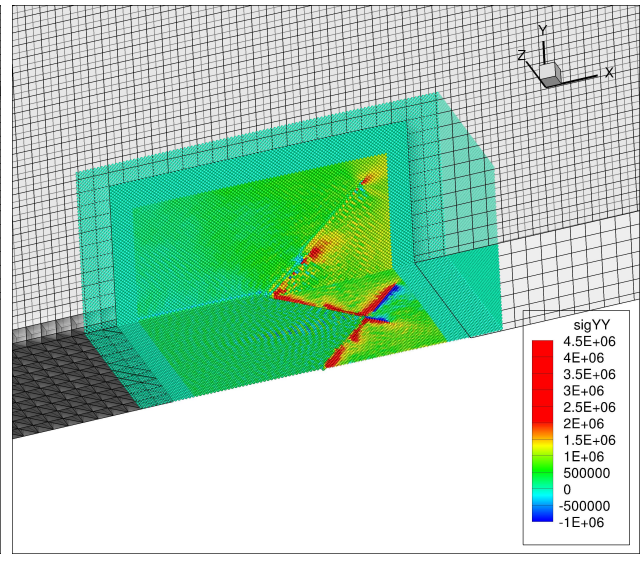

(d)

Figure 8: The virial stress contours of the cross section of the specimen in different time steps (a) step 10,000, (b) step 33,000, (c) step 34,000, (d) step 35,800. 
simulation.

Future work includes:

- performing simulations back to back with a fully atomistic model, for small problems where this is possible. In particular it will be worthwhile to investigate the role of defects in the atomistic lattice on crack propagation;

- fine-graining methods based on (goal-oriented) error estimates, allowing to spawn atomistic regions where required (see, e.g. Oden and Vemaganti [53] and Oden and Prudhomme 54] );

- coarse-graining of the cracks into continuum cracks in an energetically consistent manner, once atomistic descriptions are no-longer required;

- decreasing the computational expense through a combination of the proposed bridging scale method with adaptive model reduction techniques for fracture, e.g. Kerfriden et al. [55, 56], Galland et al. [57], and Kerfriden et al. [58]. It would be particularly interesting to investigate these model reduction techniques coupled with domain decomposition, so as to be able to reduce subdomains where non-linearities are weak and revert to full solutions of non-reducible subdomains, where non-linearities are too strong.

\section{Acknowledgements}

The authors thank the support of the German Research Foundation (DFG). Also, the authors gratefully acknowledge the help of Dr. Amitava Moitra from Pennsylvania State University for providing the WARP code.

Stéphane Bordas and Pierre Kerfriden also thank partial funding for their time provided by:

- the EPSRC under grant EP/G042705/1 Increased Reliability for Industrially Relevant Automatic Crack Growth Simulation with the eXtended Finite Element Method.

- the European Research Council Starting Independent Research Grant (ERC Stg grant agreement No. 279578) entitled "RealTCut - Towards real time multiscale simulation of cutting in non-linear materials with applications to surgical simulation and computer guided surgery." 


\section{References}

[1] E.B. Tadmor, M. Ortiz, and R. Phillips. Quasicontinuum analysis of defects in solids. Philosophical Magazine A, 73(6):1529-1563, 1996.

[2] T. Belytschko S.P. Xiao. A bridging domain method for coupling continua with molecular dynamics. Computer Methods in Applied Mechanics and Engineering, 193:1645-1669, 2004.

[3] Rateau G. Ben Dhia H. The arlequin method as a flexible engineering design tool. International Journal for Numerical Methods in Engineering, 62:1442-1462, 2005.

[4] Y. Zhang, T.J.R. Hughes, and C.L. Bajaj. An automatic 3d mesh generation method for domains with multiple materials. Computer methods in applied mechanics and engineering, 199(5):405-415, 2010.

[5] JB Cavalcante Neto, P.A. Wawrzynek, M.T.M. Carvalho, L.F. Martha, and A.R. Ingraffea. An algorithm for three-dimensional mesh generation for arbitrary regions with cracks. Engineering with Computers, 17(1):75-91, 2001.

[6] I. Babuška, G. Caloz, and J.E. Osborn. Special finite element methods for a class of second order elliptic problems with rough coefficients. SIAM Journal on Numerical Analysis, pages 945-981, 1994.

[7] T. Strouboulis, I. Babuska, and K. Copps. The design and analysis of the generalized finite element method. Computer methods in applied mechanics and engineering, 181(1-3):43-69, 2000.

[8] T. Belytschko and T. Black. Elastic crack growth in finite elements with minimal remeshing. International Journal for Numerical Methods in Engineering, 45(5):601-620, 1999.

[9] I. Babuška and J. M. Melenk. The partition of unity method. 40(4):727-758, 1997.

[10] C.A. Duarte and J.T. Oden. An hp adaptive method using clouds. Computer Methods in Applied Mechanics and Engineering, 139(1-4):237-262, 1996.

[11] R. Simpson and J. Trevelyan. A partition of unity enriched dual boundary element method for accurate computations in fracture mechanics. Computer Methods in Applied Mechanics and Engineering, 200(1-4):1-10, 2011. 
[12] R. Simpson and J. Trevelyan. Evaluation of j1 and j2 integrals for curved cracks using an enriched boundary element method. Engineering Fracture Mechanics, 2010.

[13] G. Ventura, JX Xu, and T. Belytschko. A vector level set method and new discontinuity approximations for crack growth by efg. International Journal for Numerical Methods in Engineering, 54(6):923-944, 2002.

[14] T. Rabczuk and G. Zi. A meshfree method based on the local partition of unity for cohesive cracks. Computational Mechanics, 39(6):743-760, 2007.

[15] T. Rabczuk and T. Belytschko. Cracking particles: A simplified meshfree method for arbitrary evolving cracks. International Journal for Numerical Methods in Engineering, 61 (13):2316-2343, 2004.

[16] M. Duflot and H. Nguyen-Dang. A meshless method with enriched weight functions for fatigue crack growth. International Journal for Numerical Methods in Engineering, 59(14): 1945-1961, 2004.

[17] E. Barbieri, N. Petrinic, M. Meo, and VL Tagarielli. A new weight-function enrichment in meshless methods for multiple cracks in linear elasticity. International Journal for Numerical Methods in Engineering, 2011.

[18] R. Gracie, J. Oswald, and T. Belytschko. On a new extended finite element method for dislocations: Core enrichment and nonlinear formulation. Journal of the Mechanics and Physics of Solids, 56(1):200-214, 2008.

[19] R. Gracie and T. Belytschko. An adaptive concurrent multiscale method for the dynamic simulation of dislocations. International Journal for Numerical Methods in Engineering, 2011.

[20] Ted Belytschko Mei Xu. Conservation properties of the bridging domain method for coupled molecular/continuum dynamics. International Journal for Numerical Methods in Engineering, 76:278-294, 2008.

[21] R. Gracie and T. Belytschko. Concurrently coupled atomistic and xfem models for dislocations and cracks. International Journal for Numerical Methods in Engineering, 78(3): 354-378, 2009. 
[22] VB Shenoy, R. Miller, EB Tadmor, D. Rodney, R. Phillips, and M. Ortiz. An adaptive finite element approach to atomic-scale mechanics-the quasicontinuum method. Arxiv preprint cond-mat/9710027, 1997.

[23] R.E. Miller and E.B. Tadmor. The quasicontinuum method: Overview, applications and current directions. Journal of Computer-Aided Materials Design, 9(3):203-239, 2002.

[24] R.d. Borst P. Aubertin, J. Rethor. A coupled molecular dynamics and extended finite element method for dynamic crack propagation. International Journal for Numerical Methods in Engineering, 81:72-88, 2009.

[25] R.d. Borst P. Aubertin, J. Rthor. Energy conservation of atomistic/continuum coupling. Internation Journal for Multiscale Computational Engineering, 78:1365-1386, 2009.

[26] T. Belytschko R. Gracie. An adaptive concurrent multiscale method for the dynamic simulation of dislocations. Internation Journal for Multiscale Computational Engineering, In press, 2011.

[27] FF Lange. The interaction of a crack front with a second-phase dispersion. Philosophical Magazine, 22(179):983-992, 1970.

[28] K. Ravi-Chandar and WG Knauss. An experimental investigation into dynamic fracture: Ii. microstructural aspects. International Journal of Fracture, 26(1):65-80, 1984.

[29] A. Aghaei, MJ Abdolhosseini Qomi, MT Kazemi, and AR Khoei. Stability and sizedependency of cauchy-born hypothesis in three-dimensional applications. International Journal of Solids and Structures, 46(9):1925-1936, 2009.

[30] C. Pozrikidis. On the applicability of the cauchy-born rule. Computational Materials Science, 46(2):438-442, 2009.

[31] J.M. Haile. Molecular dynamics simulation: elementary methods. John Wiley \& Sons, Inc., 1992.

[32] D.C. Rapaport. The art of molecular dynamics simulation. Cambridge Univ Pr, 2004. 
[33] J.F. Molinari G. Anciaux, S.B. Ramisetti. A finite temperature bridging domain method for md-fe coupling and application to a contact problem. Computer Methods in Applied Mechanics and Engineering, In Press, 2011.

[34] Belytschko T. Guidault PA. Bridging domain methods for coupled atomistic-continuum models with 12 or h1 couplings. International Journal for Numerical Methods in Engineering, 77(11):1566-1592, 2007.

[35] Philips R. Tadmor EB, Ortiz M. Quasicontinuum analysis of defects in solids. Philosophical Magazine A, 73(6):1529-1563, 1996.

[36] S.P. Xiao T. Belytschko. Coupling methods for continuum model with molecular model. Internation Journal for Multiscale Computational Engineering, 1:115-126, 2003.

[37] T. Menouillard, J. Réthoré, A. Combescure, and H. Bung. Efficient explicit time stepping for the extended finite element method (x-fem). International Journal for Numerical Methods in Engineering, 68(9):911-939, 2006.

[38] Steve Plimpton. Atomistic Stress Simulator (WARP), 2001. URL https://icme.hpc.msstate.edu/mediawiki/index.php/Code:_WARP.

[39] S. Plimpton. Fast parallel algorithms for short-range molecular dynamics. Journal of Computational Physics, 117(1):1-19, 1995.

[40] ABAQUS 6.11 Standard user's manual. Dessault Systems, 2011.

[41] T.P. Fries. A corrected xfem approximation without problems in blending elements. International Journal for Numerical Methods in Engineering, 75:503-532, 2008.

[42] V.P. Nguyen, T. Rabczuk, S. Bordas, and M. Duflot. Meshless methods: a review and computer implementation aspects. Mathematics and Computers in Simulation, 79(3):763$813,2008$.

[43] Nose S. Constant-temperature molecular dynamics. Journal of Physics: Condensed Matter, 2:115-119, 1990.

[44] W.H. Press. Numerical recipes in FORTRAN: the art of scientific computing, volume 1. Cambridge Univ Pr, 1992. 
[45] C. Mi, D.A. Buttry, P. Sharma, and D.A. Kouris. Atomistic insights into dislocation-based mechanisms of void growth and coalescence. Journal of Mechanics Physics of Solids, 59: 1858-1871, 2011.

[46] C.L. Kelchner, SJ Plimpton, and JC Hamilton. Dislocation nucleation and defect structure during surface indentation. Physical Review B, 58(17):11085, 1998.

[47] F. Cleri, D. Wolf, S. Yip, and SR Phillpot. Atomistic simulation of dislocation nucleation and motion from a crack tip. Acta materialia, 45(12):4993-5003, 1997.

[48] Y. Sun, S. Izumi, S. Hara, and S. Sakai. Anisotropy behavior of dislocation nucleation from a sharp corner in copper. Journal of Computational Science and Technology, 5(1):54-61, 2011.

[49] L. Xiong, Q. Deng, G. Tucker, D.L. McDowell, and Y. Chen. A concurrent scheme for passing dislocations from atomistic to continuum domains. Acta Materialia, 60(3):899913, 2012.

[50] J.S. Robert. Comments on virial theorems for bounded systems. American Journal of Physics, 51:940-942, 1983.

[51] A.K. Subramaniyan and CT Sun. Continuum interpretation of virial stress in molecular simulations. International Journal of Solids and Structures, 45(14-15):4340-4346, 2008.

[52] M.J. Bühler. Atomistic modeling of materials failure. Springer Verlag, 2008.

[53] J.T. Oden and K.S. Vemaganti. Estimation of local modeling error and goal-oriented adaptive modeling of heterogeneous materials: I. error estimates and adaptive algorithms. Journal of Computational Physics, 164(1):22-47, 2000.

[54] J.T. Oden and S. Prudhomme. Estimation of modeling error in computational mechanics. Journal of Computational Physics, 182(2):496-515, 2002.

[55] P. Kerfriden, P. Gosselet, S. Adhikari, S. Bordas, and J.C. Passieux. Pod-based model order reduction for the simulation of strong nonlinear evolutions in structures: application to damage propagation. In IOP Conference Series: Materials Science and Engineering, volume 10, page 012165. IOP Publishing, 2010. 
[56] P. Kerfriden, P. Gosselet, S. Adhikari, and SPA Bordas. Bridging proper orthogonal decomposition methods and augmented newton-krylov algorithms: an adaptive model order reduction for highly nonlinear mechanical problems. Computer Methods in Applied Mechanics and Engineering, 200(5):850-866, 2011.

[57] F. Galland, A. Gravouil, E. Malvesin, and M. Rochette. A global model reduction approach for 3d fatigue crack growth with confined plasticity. Computer Methods in Applied Mechanics and Engineering, 200(5):699-716, 2011.

[58] P. Kerfriden, J.C. Passieux, and S.P.A. Bordas. Local/global model order reduction strategy for the simulation of quasi-brittle fracture. International Journal for Numerical Methods in Engineering, 89(2):154-179, 2012. 